\title{
Switching transients due to a power factor correction capacitor bank in LV power system and their comparison with lightning impulses
}

\begin{abstract}
Switching transients generated by a five-step 50 KVAR shunt capacitor bank in a 415 $\mathrm{V} / 240 \mathrm{~V}$ power system have been characterized with the view of providing a database to investigate their effects on electrical network components. Three balanced single-phase RL loads were connected to the three-phases separately. The results show that the current impulses generated during the switching operations may be high enough to either damage or degrade the equipment connected to the power system. The experimental set up was consequently simulated by means of Power System Computer-Aided Design (PSCAD) software. Data analysis were conducted on the amplitude, the rise time and the pulse duration of the transient current waveform. The results of the experimental Work and the simulation have reasonable agreement. Thus, the PSCAD simulation model could be used to generate capacitor bank switching impulses for various configurations in a low voltage system, to develop a data bank. The measured and simulated transient waveforms are compared with extreme case (higher 5\%) of positive lightning waveforms. The outcome of this study can serve as a guide for manufacturing technologists as well as electrical engineers in addressing a main issue of capacitor banks, the generation of switching transients in LV systems and their effects on downstream equipment.
\end{abstract}

Keyword: Capacitor banks; Switching transient; Power systems; Power factor; PSCAD 